\title{
Xiaoqinglong Granules as Add-On Therapy for Asthma: Latent Class Analysis of Symptom Predictors of Response
}

\author{
Qinglin Zha, ${ }^{1}$ Seqi Lin, ${ }^{1}$ Chi Zhang, ${ }^{2}$ Christopher Chang, ${ }^{3}$ Hanrong Xue, ${ }^{4}$ Cheng Lu, \\ Miao Jiang, ${ }^{2}$ Yan Liu, ${ }^{2}$ Zuke Xiao, ${ }^{5}$ Weiyou Liu, ${ }^{6}$ Yunfei Shang, ${ }^{7}$ Jianjian Chen, ${ }^{8}$ \\ Minyong Wen, ${ }^{9}$ and Aiping $\mathrm{Lu}^{2,10}$ \\ ${ }^{1}$ School of Computer, Jiangxi University of Traditional Chinese Medicine, Nanchang 330004, China \\ ${ }^{2}$ Institute of Basic Research in Clinical Medicine, China Academy of Chinese Medical Sciences, Beijing 100700, China \\ ${ }^{3}$ Division of Allergy and Immunology, Thomas Jefferson University, Wilmington, DE 19803, USA \\ ${ }^{4}$ Department of Respiratory Medicine, Affiliated Hospital of Jiangxi University of TCM, Nanchang 330004, China \\ ${ }^{5}$ Department of Respiratory Medicine, People's Hospital of Jiangxi Province, Nanchang 330006, China \\ ${ }^{6}$ Department of Respiratory Medicine, The First Affiliated Hospital of Gannan Medical University, Gannan 314000, China \\ ${ }^{7}$ Department of Respiratory Medicine, The Third Affiliated Hospital of Nantong University, Nantong 216000, China \\ ${ }^{8}$ Department of Respiratory Medicine, Nanchang Hospital of Integrated Traditional Chinese and Western Medicine, \\ Nanchang 330003, China \\ ${ }^{9}$ Department of Respiratory Medicine, The First Affiliated Hospital of Guangzhou University of TCM, Guangzhou 510405, China \\ ${ }^{10}$ School of Chinese Medicine, Hong Kong Baptist University, Kowloon Tong, Kowloon, Hong Kong
}

Correspondence should be addressed to Aiping Lu; lap64067611@126.com

Received 30 July 2012; Accepted 3 December 2012

Academic Editor: Haruki Yamada

Copyright (C) 2013 Qinglin Zha et al. This is an open access article distributed under the Creative Commons Attribution License, which permits unrestricted use, distribution, and reproduction in any medium, provided the original work is properly cited.

\begin{abstract}
Xiaoqinglong granules (XQLG) has been shown to be an effective therapy in asthma animal models. We reviewed the literature and conducted this study to assess the impact of XQLG as an add-on therapy to treatment with fluticasone/salmeterol (seretide) in adult patients with mild-to-moderate, persistent asthma. A total of 178 patients were randomly assigned to receive XQLG and seretide or seretide plus placebo for 90 days. Asthma control was assessed by asthma control test (ACT), symptoms scores, FEV ${ }_{1}$, and PEF. Baseline patient-reported Chinese medicine (CM)-specific symptoms were analyzed to determine whether the symptoms may be possible indicators of treatment response by conducting latent class analysis (LCA). There was no statistically significant difference in ACT score between two groups. In the subset of 70 patients with symptoms defined by CM criteria, XQLG add-on therapy was found to significantly increase the levels of asthma control according to global initiative for asthma (GINA) guidelines $(P=0.0329)$. There was no significant difference in another subset of 100 patients with relatively low levels of the above-mentioned symptoms $(P=0.1291)$. Results of LCA suggest that patients with the six typical symptoms defined in CM may benefit from XQLG.
\end{abstract}

\section{Introduction}

Complementary or alternative medicine (CAM) is extensively used in the treatment of asthma. In spite of the multitude of Western medicine treatments available for patients with asthma [1-4], only $41 \%$ of UK asthma sufferers have not used CAM and of those two-thirds would consider using it in the future [5]. Recommendations for the use of CAMs should be based only on rigorous proof of efficacy derived from high-quality studies [6]. Chinese herbal medicines (CHMs) are a consistently popular form of CAMs in asthma and proprietary asthma drugs are derived from herbal remedies [7]. Some of these herbal remedies have a millenary history and represent the traditional medicine in many countries. Xiaoqinglong decoction (XQLD), also known as Sho-seiryuto (SST) in Japan and So-cheong-ryong-tang (SCRT) in Korea, was first written about by Zhang Zhong Jing, in the Traditional Chinese Medicine (TCM) classic text Shang Han 
TABLE 1: Components of Xiaoqinglong granule (with sugar, $1000 \mathrm{~g})^{*}$ and their chinese medicine treatment functions.

\begin{tabular}{|c|c|c|c|c|}
\hline Pi yin name & Latin name & Part used & Weight & Chinese medicine treatment functions \\
\hline Ma Huang & Ephedra sinica Stapf. & Stems & $154 \mathrm{~g}$ & $\begin{array}{l}\text { Ma Huang resolves the exterior and diffuses the lungs, stops } \\
\text { coughing, and levels panting. }\end{array}$ \\
\hline Gui Zhi & $\begin{array}{l}\text { Cinnamomum cassia } \\
\text { Presl. }\end{array}$ & Twigs and branches & $154 \mathrm{~g}$ & $\begin{array}{l}\text { Gui Zhi resolves the exterior and scatters cold. When } \\
\text { combined with Ma Huang, Gui Zhi strengthens Ma Huang's } \\
\text { function of promoting diaphoresis. }\end{array}$ \\
\hline Bai Shao & Paeonia lactiflora Pall. & Roots & $154 \mathrm{~g}$ & Bai Shao is used to harmonize the blood to regulate qi. \\
\hline Gan Jiang & Zingiber officinale Rosc. & Rhizomes & $154 \mathrm{~g}$ & Gan Jian scatter cold, dispel dampness. \\
\hline $\mathrm{Xi} \mathrm{Xin}$ & $\begin{array}{l}\text { Asarum heterotropoides } \\
\text { Fr. Schm. }\end{array}$ & Whole & $77 \mathrm{~g}$ & Xi Xin prevents wind cold. \\
\hline Zhi Gan Cao & $\begin{array}{l}\text { Glycyrrhiza uralensis } \\
\text { Fisch. }\end{array}$ & Roots & $154 \mathrm{~g}$ & $\begin{array}{l}\text { Mix-fried Gan Cao harmonizes the other medicinal in this } \\
\text { formula. }\end{array}$ \\
\hline Fa Ban Xia & $\begin{array}{l}\text { Pinellia ternate (Thunb.) } \\
\text { Breit. }\end{array}$ & Rhizomes & $231 \mathrm{~g}$ & Ban Xia transforms phlegm and down bears the qi. \\
\hline Wu Wei Zi & $\begin{array}{l}\text { Schisandra chinensis } \\
\text { (Turcz.) Baill. }\end{array}$ & Fruits & $154 \mathrm{~g}$ & $\begin{array}{l}\text { Wu Wei Zi engenders fluids, constrains the lung qi, and helps } \\
\text { stabilize panting. }\end{array}$ \\
\hline
\end{tabular}

Chinese Pharmacopoeia (2005) [12].

Lun (Treatise on Cold Damage Diseases), about 1,800 years ago $[8,9]$. There are eight herb components in the medicine. These constituents and their actions are described in Table 1 $[10,11]$.

XQLD is a popular intervention which has been used for the treatment of asthma triggered by upper respiratory infections [13]. It is most suitable when phlegm is excessive, clear, and watery and the body feels chilly $[14,15]$. Accordingly, XQLD is not used to treat dry cough or cough with thick or yellow phlegm [11]. Since TCM was brought to Japan and many other Asian countries [16], XQLD has had a very great influence on the practice of medicine. A large sample-sized retrospective study indicates that XQLD is a very commonly used formula in Taiwan $[8,17]$. XQLD has attracted Asian scientists with an interest in investigating mechanisms of action since the 1980s, particularly in Taiwan [18] and Japan [19].

1.1. A Review of the Pharmacologic and Clinical Effects of $X Q L D$. The general pharmacological properties of XQLD extracts were investigated in various experimental animals [20]. XQLD has been reported to show antiallergic effects [21-23] and appears to be useful for treating type I allergic reactions. There are few antihistaminic side effects, such as the sedation and drowsiness that result from blockade of histamine $\mathrm{H} 1$ and muscarinic receptors in the brain [24]. The suppressive activity by XQLD on chemical mediators, such as histamine and leukotrienes from peritoneal mast cells in vitro, has also been demonstrated [23].

A previous study in guinea pigs demonstrated that the antiasthmatic effects of XQLD appeared to be partly mediated by stimulation of $\beta 2$-adrenoceptors, leading to broncho relaxation, and that XQLD inhibits the infiltration of eosinophils into the airway [25]. Fresh data on asthma has shown that at least a part of the mechanism of action of XQLD against OVA-sensitized allergic airway inflammation in a mouse model is different from that of prednisolone, and the results demonstrate that XQLD alleviates nasal symptoms by the inhibition of histamine signaling through suppression of TDI-induced H1R and HDC gene upregulation [19].

In addition, due to the multiple components in XQLD, there may be other mechanisms of action. For example, XQLD appears to inhibit mast cell degranulation [26] and influence CD4(+) $\mathrm{T}$ cells, which may be significant in developing its use in the treatment of IgE-mediated allergic asthma [16]. The role that XQLD may play in the regulation of cytokines and chemokines and the subsequent effect on allergic airway inflammation is not known [27]. Kao and colleagues demonstrated that XQLT exhibits antiairway inflammatory, anti-airway remodeling, and specific immunoregulatory effects in a chronic asthmatic mice model [28]. Their results indicated that XQLD treatment alleviates asthma-like pulmonary inflammation via suppression of specific chemokines [29]. Since many physicians frequently prescribe XQLD in combination with conventional Western medicine, researchers evaluated the pharmacokinetic interactions in rats to evaluate the potential for adverse effects of the combined therapy $[30,31]$.

Similarly, according an experimental study from Japan, authors examined whether XQLD administration is affected by a probiotic product and concluded that XQLD-mediated anti-allergic effects are enhanced by the probiotic [32]. The XQLD constituent herbs, including Herba Ephedrae and Herba Asari, have been reported to have cytokinemodulating effects [33]. Accordingly, XQLD has been used not only for "symptomatic" treatment of allergic diseases but also for "prevention." It is important to note that the pharmacokinetics of the ephedrine component of XQLD has also been investigated $[34,35]$.

Previous clinical trials of XQLD were nonrandomized or randomized to include a standard of care control group. In these initial studies, XQLD was associated with a decrease in the long-term rate of mild asthma exacerbations, improvement in asthma control, and improvements in lung function 
[13]. A previous clinical trial has also demonstrated its potential benefits in improving quality of life in asthmatics [36]. Unfortunately, most of the previous clinical trials on XQLD are of poor methodological quality and therefore XQLD remains of uncertain value in the treatment of asthma [13]. Since XQLD remains popular for the treatment of asthma in Asian communities, this study was conducted to further elucidate the efficacy and safety of XQLD in the treatment of asthma.

1.2. The Current Status of XQLD Research in Asthma. To our knowledge, this is the largest randomized positive-controlled trial to test XQLD in the treatment of asthma. Trial designers were concerned about standardized intervention because many of TCM decoctions cannot ensure reproducibility of therapeutic effects. Xiaoqinglong granules (XQLG), a Chinese patent medicine of the same name as XQLD, has the same proportions of ingredients and is manufactured in accordance with the Chinese Pharmacopoeia [12]. In Japan, most uses of granules are based upon classical formulas. The vast majority of the formulas from the Shang Han Lun are particularly prominent in modern clinical use. Granules have also become deeply integrated into healthcare in Taiwan over the past 30 years [37]. This development was largely stimulated by the convenience of granules for patients, along with the fact that the governments of both Japan and Taiwan also streamlining the process for applications exclusively when granules products apply for classical formulas coverage [38]. This has spurred a tremendous amount of growth and economic investment, as well as widespread clinical use and the experience that comes with increased use. Granules are desirable for large scale healthcare distribution because they are well regulated and hygienic, and they are ideal for research because batches are consistent and traceable.

Likewise, the Chinese government maintains the registration administration mechanism that oversees TCM combination preparations originating from historic, classic, and well-known recipes. The application data for marketing TCM combination preparations may be partially exempted based on formulation and source as well as their composition, function, indications, and manufacturing procedures [39]. However, perhaps as a result of this streamlining of the process for phase I, II, and III trials, there are certain barriers to obtaining more specific indications and detailed safety assessment reports of current products. High quality research is needed in the area of efficacy and safety of traditional Chinese herbal medications.

1.3. Chinese Medicine Patterns of Asthma. The aim of the current study, therefore, is precisely to determine the effectiveness and safety of XQLG for the treatment of asthma in adults. In addition, the predictive value of the baseline characteristics with respect to response to the therapy is investigated due to the importance of clinical characteristics, as reported in previous studies. Similarly, Chinese medicine (CM) symptoms are very important for $\mathrm{CM}$ diagnostics in daily clinical practice. In CM, a specific set of symptoms is referred to as a pattern. CM pattern classification has been used to define subsets of patients within a few thousand years. This is analogous to the development of personalized medicine in Western medicine concepts, where active research is being conducted to define genetic or nongenetic characteristics that predict treatment response to new or existing drugs. The additional specific study aims are therefore to (1) use latent class analysis, a statistical model to classify asthma adults with symptoms predicators; (2) provide evidence of latent class concurrent validity by examining association between class membership and unmet healthcare needs.

\section{Methods}

2.1. Patients. Male and female patients (aged 18-65 year) with a documented history of asthma as defined by the National Institute of Health (NIH) [40] and Global Initiative for Asthma (GINA) [41] were eligible for study entry. Other inclusion criteria were reversibility of baseline forced expiratory volume in one second $\left(\mathrm{FEV}_{1}\right)(\geq 15 \%$ and $\geq 200 \mathrm{~mL})$ in response to inhaled salbutamol, and "asthma of cold pattern" according to Criteria of Diagnosis and Therapeutic Effect of Disease and Syndromes in Traditional Chinese Medicine [42]. According to the guidelines of asthma treatment and prevention in China (GATPC) [43], patients are divided into three types, including acute exacerbation, chronic persistent, and clinical remission. This trial included only participants with mild-to-moderate persistent asthma.

Exclusion criteria included the following. Contraindicated medications included theophylline, corticosteroids, and short-acting beta 2 agonist (SABA) therapy within the past two weeks; any significant disease or disorder that may influence results or a patient's ability to participate in the study; "asthma of heat pattern" according to Criteria of Diagnosis and Therapeutic Effect of Disease and Syndromes in Traditional Chinese Medicine [42]; women who were pregnant or breastfeeding; known hypersensitivity to any intervention; participation in other investigational studies in the last 4 weeks.

The study was conducted in accordance with the Declaration of Helsinki and was approved by local ethics committees and institutional review boards as appropriate. All patients provided written informed consent prior to participating in the study.

2.2. Study Design. A randomized, double-blind, positive controlled, parallel-group, multicenter study was conducted at the six centers in China. The study, focusing on a superiority study design, was sequentially conducted as follows: a runin period of one week prior to randomization, followed by a treatment period of 90 days. At the end of the run-in period, participants were randomized to the active XQLG/seretide group (Group A) or placebo/seretide group (Group B) by the central randomization system (Figure 1). This system is provided by China Academy of Chinese Medical Sciences, which adopted the computer telephone integration (CTI) technology to integrate computers, internet, and telecom. The random number list is assigned by interactive voice response (IVR) and interactive web response (IWR) [44]. The success 


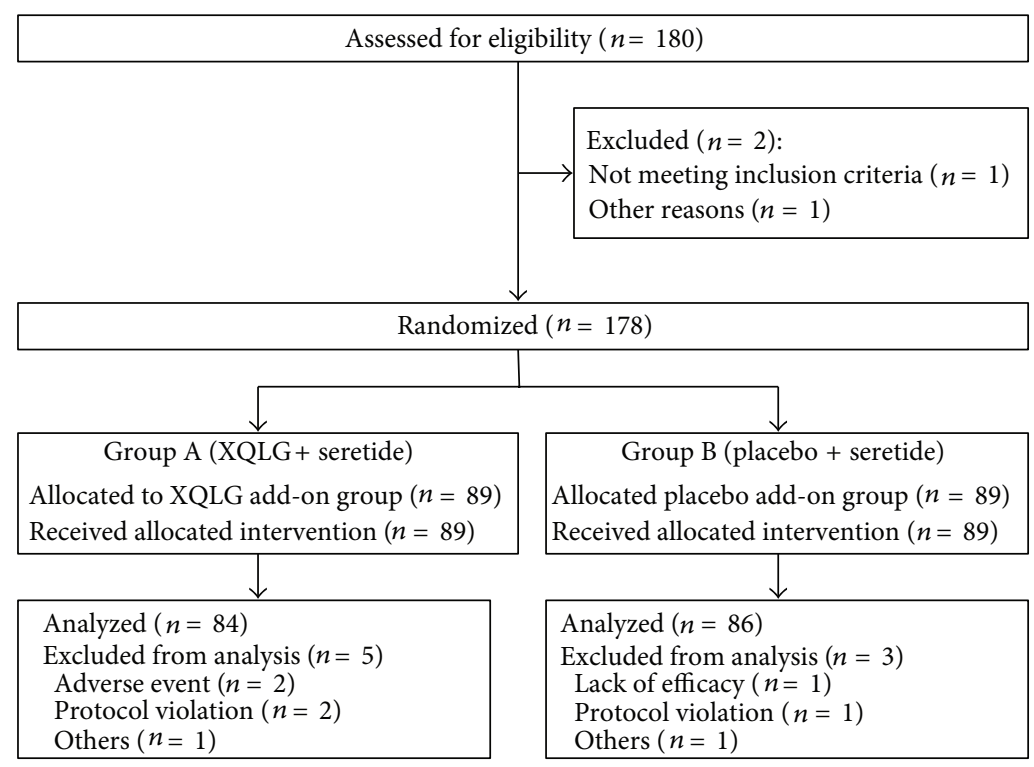

FIGURE 1: Flow Diagram of the enrollment progress through the phases of XQLG randomized trial of the two groups.

of blinding will be assessed at each participant's last visit. An assessor who did not participate in the treatment and who is blinded to the allocation results will perform the outcome assessment.

Sample size calculation was projected with the use of SAS software. Based on our pilot trial, the response rate after 90 days was estimated at $75 \%$ in the conventional treatment group and $94 \%$ in XQLG add-on therapy group (level of significance $5 \%$, power of $90 \%$ ).

\section{Treatment}

\section{1. $X Q L G$}

Proprietary name: Xiaoqinglong granules.

Chinese pronunciation: Xiaoqinglong Keli.

Xiaoqinglong granules (State Food and Drug Administration (China) Approval no. Z19993076) used was manufactured by Beijing Shouer Pharmaceutical Factory (Beijing, China) (batch no. 091201).

Ingredients: Herb Ephedrae, Ramulus Cinnamomi, Rhizoma Zingiberis, Radix Asari, Fructus Schisandrae, and Radix Paeoniae Alba (Table 1).

Quality control: Beijing Shouer Pharmaceutical Factory is a GMP certificated pharmaceutical factory. Its product (batch no. 091201) complies with the requirements of Chinese Pharmacopoeia 2005. According to pharmacopeia requirement, compounds Ephedrine hydrochloride, Pseudoephedrine hydrochloride, and Paeoniflorin in Xiaoqinglong granules sample were determined $(6.232 \mathrm{mg} / \mathrm{g}$, $2.853 \mathrm{mg} / \mathrm{g}$, and $0.8538 \mathrm{mg} / \mathrm{g}$, resp.) and fully comply with Chinese pharmacopeia 2005 standard. Identification details refer to Chinese Pharmacopoeia (2005 edition).
Description: light brown to brown granule, or grey to light brown, smells slightly fragrant, tastes sweet and slightly pungent.

3.2. Seretide. Seretide is a combination medication containing an inhaled corticosteroid (fluticasone) and a long acting reliever or symptom controller (salmeterol). This combination of salmeterol xinafoate and fluticasone propionate is recommended by GINA as a regular treatment for uncontrolled asthma [41].

Subjects in the XQLG add-on group received oral XQLG (taken after being dissolved in boiled water, 13 grams/1 pack, three times a day) and seretide (1 puffs twice daily). Subjects in the seretide group received xiaoqinglong placebo granules for 90 days and seretide (1 puffs twice daily).

Subjects requiring additional intervention at any time because of disease severity were withdrawn from the study. For the duration of the study, inhaled Ventolin (100 to $200 \mu \mathrm{g})$ 3 to 4 times daily, not exceeding 8 puffs $(800 \mu \mathrm{g} /$ day $)$, was allowed as needed.

3.3. Clinical and Laboratory Evaluation. The primary outcomes were the achievement of asthma control and asthma control evolution. Asthma control achievement was based on clinical control (as recommended by GINA 2006) [41, 45]. Asthma control evolution was measured with the ACT (Dutch version). The ACT is a clinically validated measure for asthma control, consisting of five questions, each having five possible response modalities (classified by decreasing levels of asthma control, scored from 5 to 1$)[46,47]$. The ACT score (range 5-25) was determined by summing the response scores to the five questions; the higher the score, the better the asthma control. The ACT was filled out by patients at the start of the run-in phase, at randomization and 7, 30, 60, and 90 days after randomization. 
TABLE 2: Two latent class models of asthma participants and relative symptoms response probabilities.

\begin{tabular}{lcc}
\hline Item types (symptom) & $\begin{array}{c}\text { Rule 1 } \\
\text { Class 1 subset } \\
(n=100)\end{array}$ & $\begin{array}{c}\text { Rule 2 } \\
\text { Class 2 } \\
\text { subset } \\
(n=70)\end{array}$ \\
\hline Qi vacuity & $\mathbf{0 . 8 9 0 3}{ }^{\dagger}$ & $\mathbf{1 . 0 0 0 0}$ \\
Chest tightness & $\mathbf{0 . 7 7 3 5}$ & $\mathbf{0 . 9 6 7 2}$ \\
Aversion to cold & $\mathbf{0 . 6 3 8 5}$ & $\mathbf{0 . 7 4 5 4}$ \\
Clear, pale, or watery phlegm & 0.3927 & $\mathbf{0 . 9 9 8 4}$ \\
Torpid intake & 0.3554 & $\mathbf{0 . 6 9 3 5}$ \\
Coughing & $\mathbf{0 . 8 6 0 4}$ & $\mathbf{1 . 0 0 0 0}$ \\
Fever & 0.0000 & 0.0287 \\
No thirst & $\mathbf{0 . 7 4 8 1}$ & $\mathbf{0 . 4 5 8 8}$ \\
\hline Latent class prevalence & 0.5898 & 0.4102 \\
\hline
\end{tabular}

Note. The model shows the different response patterns of the two rules on different item types. Rule 1; Rule 2.

${ }^{\dagger}$ Item-response probabilities $>.5$ in bold to facilitate interpretation. Itemresponse probabilities corresponding to a yes response; Qi vacuity: shortness of breath; chest tightness: oppression in the chest, feeling of oppression in the chest; aversion to cold: sensation of cold which cannot be relieved by warmth, also known as chills; clear, pale, or watery phlegm; torpid intake: loss of appetite with no desire for food with decreased intake, the same as poor appetite; coughing: (1) the expelling of air from the lungs suddenly with an explosive noise or expectoration of sputum; (2) any disease mainly manifested by cough; fever: elevation of the body temperature above the normal or subjective feeling of feverishness; no thirst: no feeling of dryness of the mouth with a desire to drink.

Secondary outcomes included changes in lung function pre- and posttests $\left(\mathrm{FEV}_{1}, \mathrm{PEF}\right)$. Asthma symptom scores, measured on a scale of 0 to $6(0=$ no symptoms; $2=$ mild symptoms; $4=$ moderate symptoms; $6=$ severe symptoms), were also recorded. These scores were summed to obtain the total daily asthma symptom score in 7, 30, 60, 90 days. Safety evaluations included incidence of treatment-emergent adverse events (AEs) and serious adverse events (SAEs).

3.4. Statistical Analysis. All randomized subjects were assessed by comparing baseline characteristics of both study groups using unpaired $t$-tests for continuous variables, and chi-squared tests or Wilcoxon rank sum test for categorical variables. The primary analysis was performed on the intent-to-treat (ITT) approach including all randomized patients with a baseline value and at least 1 treatment period measurement. The ACT scores measured at baseline and 90 days were analyzed using a repeated measures multivariate ANOVA. Asthma control (GINA 2006) was analyzed using Wilcoxon rank sum test at endpoint. Missing data for secondary endpoints were imputed using the Last Observation Carried Forward method. Unless otherwise stated, results are reported as mean $\pm \mathrm{SD}$. Assumptions of normality and homoscedasticity were assessed. All statistical tests were 2 tailed, and $P$ values less than .05 were considered statistically significant. The safety evaluation included all randomized patients. The number and percentage of patients reporting clinical adverse experiences were summarized by treatment group.
TABLE 3: Subject demographics and baseline characteristics (ITT population).

\begin{tabular}{lcc}
\hline & $\begin{array}{c}\text { XQLG add-on } \\
\text { group }(n=84)\end{array}$ & $\begin{array}{c}\text { Placebo add-on } \\
\text { group }(n=86)\end{array}$ \\
\hline $\begin{array}{l}\text { Age, year, mean (SD) } \\
\text { Sex, } n \text { male (\%) }\end{array}$ & $44.13(12.42)$ & $44.20(11.92)$ \\
BMI, kg/m ${ }^{2}$, mean (SD) & $22.66(3.22)$ & $22.72(2.83)$ \\
GATPC & & \\
$\quad$ II & $45(53.57)$ & $44(51.16)$ \\
$\quad$ III & $39(46.43)$ & $42(48.84)$ \\
$\begin{array}{l}\text { Duration of disease, year, } \\
\text { mean (minimum, }\end{array}$ & $10.83(0.00,52.00)$ & $11.24(0.00,54.00)$ \\
maximum) & & \\
FEV, (\%), mean (SD) & $75.60(25.91)$ & $72.45(22.61)$ \\
PEF, L/min, mean (SD) & $282.71(135.84)$ & $282.31(20.92)$ \\
ACT, mean score (SD) & $16.86(3.67)$ & $16.94(3.57)$ \\
\hline
\end{tabular}

Definition of abbreviations: GATPC: guideline of asthma treatment and prevention in China; ACT: asthma control test; ITT: intent-to-treat.

Given the identical protocols and measurements, baseline data for Group A (Figure 1) and Group B clinical studies were combined for the subset analyses. The latent classes were determined based on presence or absence of 8 symptoms: Qi vacuity, chest tightness, aversion to cold, clear, pale, or watery phlegm, torpid intake, coughing, fever, and no thirst. Table 2 presents each of the variables and how they were categorized.

In order to examine the structure underlying the set of 8 symptoms, latent class analysis (LCA) was performed using SAS PROC LCA (SAS version 9.2, Cary, NC, USA: SAS Institute Inc.) [48]. LCA is a statistical method that is used to classify subtypes of patients according to selected TCM clinical characteristics and estimate the appropriate number of latent classes based on the lower Bayesian information criteria (BIC) value and the Akaike information criterion (AIC). In PROC LCA, missing data are managed by maximum likelihood using an expectation-maximization (EM) procedure, with data assumed to be missing at random.

\section{Results}

A total of 178 patients underwent randomization, of which 170 patients comprised the ITT population. Reasons for patient withdrawal at the screening and randomization stages are summarized in Figure 1. Nearly half of the patients were men (47.6\%), and the age ranged from 19 to 65 year. Baseline demographics and clinical characteristics were well matched between groups (Table 3). The subjects enrolled in this trial had inadequately controlled asthma as defined by the inclusion criteria of the study design and a low ACQ score consistent with poorly controlled asthma.

4.1. Asthma Outcomes. Compared with baseline, the improvements in uncontrolled levels of asthma control according to GINA guidelines during the 90-day treatment period were observed both in the XQLG $13 \mathrm{~g}$ three times daily combination therapy and control groups (uncontrolled (\%) 
TABLE 4: Assessment of Level of asthma control according to GINA Guidelines.

\begin{tabular}{|c|c|c|c|c|}
\hline Visit & Levels of asthma control & XQLG add-on group $(n, \%)$ & Placebo add-on group $(n, \%)$ & $P$ value \\
\hline \multirow{5}{*}{0 day } & Uncontrolled & $76(90.48)$ & $80(93.02)$ & \multirow{3}{*}{0.5470} \\
\hline & Partly controlled & $8(9.52)$ & $6(6.98)$ & \\
\hline & Controlled & $0(0.00)$ & $0(0.00)$ & \\
\hline & Total & 84 & 86 & \\
\hline & Uncontrolled & $5(6.58)$ & $9(11.25)$ & \multirow{3}{*}{0.8883} \\
\hline \multirow[t]{3}{*}{ 90th day } & Partly controlled & $41(53.95)$ & $38(47.50)$ & \\
\hline & Controlled & $30(39.47)$ & $33(41.25)$ & \\
\hline & Total & 76 & 80 & \\
\hline
\end{tabular}

TABLE 5: Asthma control test (ACT) score and asthma symptoms score in trial visit.

\begin{tabular}{lclc}
\hline & Day & $\begin{array}{l}\text { Difference in mean change } \\
\text { Group A versus Group B (95\% CI) }\end{array}$ & $P$ value \\
\hline \multirow{4}{*}{ ACT Score } & 70 & $0.26(-1.31$ to 1.82$)$ & 0.5914 \\
& 60 & $0.65(-1.19$ to 2.49$)$ & 0.7166 \\
& 90 & $0.35(-1.49$ to 2.19$)$ & 0.2485 \\
Asthma & 7 & $-1.09(-3.42$ to 1.24$)$ & 0.5334 \\
symptoms & 30 & $-0.68(-3.39$ to 2.02$)$ & 0.1255 \\
& 60 & $-1.42(-4.1$ to 1.25$)$ & 0.4083 \\
& 90 & $-1.13(-3.83$ to 1.58$)$ & 0.0823 \\
\hline
\end{tabular}

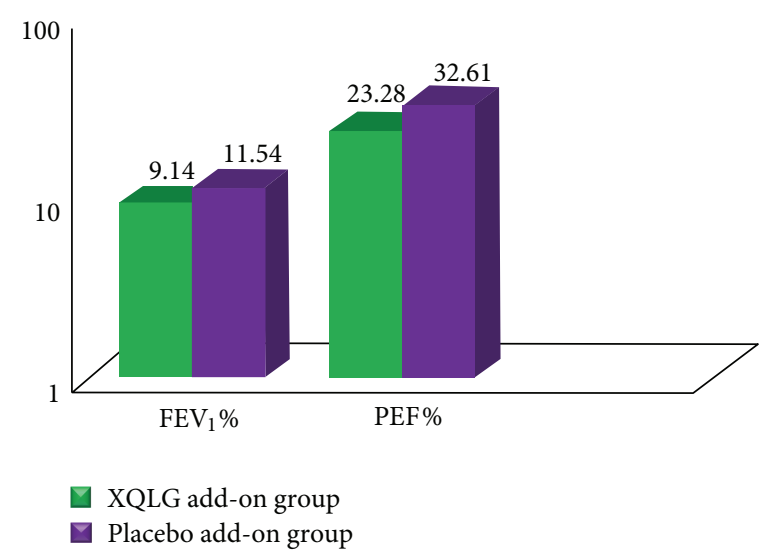

FIGURE 2: Adjusted mean change from baseline in forced expiratory volume $\left(\mathrm{FEV}_{1} \%\right)$ and peak expiratory flow $(\mathrm{PEF} \%)$ during the entire 90-day treatment period.

from 90.48 to 6.58 , from 93.02 to 11.25 , resp.). However, no statistically significant difference between treatment groups was apparent ( $P$ values 0.8883 ) (Table 4$)$, whilst significant amelioration of the predefined scores was seen in both groups. Similarly, although all treatment groups demonstrated a mean improvement in ACT score and asthma symptoms (Table 5), between-treatment differences were also not statistically significant. Estimated mean differences were 0.35 (95\% CI, -1.49 to $2.19 ; P=0.5334)$ and $-1.13(95 \%$ $\mathrm{CI},-3.83$ to $1.58 ; P=0.1728)$, respectively, for 90 -day evaluation of ACT Score and Asthma Symptoms. Overall, the improvements in ACT score and asthma symptoms with
TABLE 6: Subject demographics and baseline characteristics of class 1 and 2 subset $(n=170)$.

\begin{tabular}{lccc}
\hline & $\begin{array}{c}\text { Class 1 subset } \\
(n=100)\end{array}$ & $\begin{array}{c}\text { Class 2 subset } \\
(n=70)\end{array}$ & $P$ value \\
\hline Age, year, mean (SD) & $43.67 \pm 12.61$ & $44.87 \pm 11.46$ & 0.5462 \\
Sex, $n$ male (\%) & $51(55.00)$ & $29(41.43)$ & 0.2199 \\
BMI, kg/m ${ }^{2}$, mean (SD) & $22.86 \pm 3.39$ & $22.46 \pm 2.40$ & 0.4990 \\
GATPC, mean score (SD) & & & \\
$\quad$ II & $53(53.00)$ & $36(51.43)$ & 0.8405 \\
$\quad$ III & $47(47.00)$ & $34(48.57)$ & \\
$\begin{array}{l}\text { Duration of disease, year, } \\
\text { mean (SD) }\end{array}$ & $12.38 \pm 13.74$ & $9.13 \pm 10.52$ & 0.2448 \\
FEV $(\%)$, mean (SD) & $72.76 \pm 26.52$ & $75.84 \pm 20.73$ & 0.7313 \\
PEF, L/min, mean (SD) & $287.6 \pm 140.07$ & $275.3 \pm 117.32$ & 0.4194 \\
\hline
\end{tabular}

XQLG were paralleled by significant improvements in control group scores for 7 days, 30 days, 60 days, and 90 days (all $P>0.05)$.

Throughout the study period, the mean change from baseline in $\mathrm{FEV}_{1} \%$ increased for all treatment groups; at the end of the 90-day treatment period, $\mathrm{FEV}_{1} \%$ increased to $9.14 \%$ in XQLG add-on therapy and $11.54 \%$ for seretide. There was no statistically significant difference between the groups (Figure 2). The mean improvement in PEF\% showed no between-treatment differences between the XQLG and control groups.

LCA grouped all individuals into 2 classes (groups) according their symptoms. To determine the presence of latent classes for symptoms, 1 and 2 class models were tested. As shown in Table 2, item-response probabilities $>0.5$ in bold were defined to facilitate interpretation in a 2-class model. Class 1 consisted of $58.98 \%(n=100)$ of the sample. As can be seen in Figure 3, the symptoms trend of adherence was similar in both classes. These participants were consistent in their responses to cold pattern symptoms. Class 2 consisted of the remainder $41.02 \%(n=70)$ of the sample. Compared with participants assigned to class 1 , class 2 participants had a higher probability of reporting adherence to the six typical symptoms, including Qi vacuity; chest tightness; aversion to cold; clear, pale, or watery phlegm; torpid intake; coughing. Conditional probabilities are provided in Table 2 and represented in Figure 3. Analyses were conducted to compare characteristics of class 1 to class 2 participants (Table 6). Duration of disease, age, gender, $\mathrm{FEV}_{1}$ (\%), or PEF 
TABLE 7: Assessment of levels of asthma control according to GINA guidelines in class 1 and class 2 subset of participants.

\begin{tabular}{|c|c|c|c|c|c|c|c|c|c|c|}
\hline & \multicolumn{3}{|c|}{0 day } & \multirow{2}{*}{$\begin{array}{l}\text { Mean } \\
\text { score }\end{array}$} & \multirow[b]{2}{*}{$P$ value } & \multicolumn{3}{|c|}{ 90th day } & \multirow{2}{*}{$\begin{array}{l}\text { Mean } \\
\text { score }\end{array}$} & \multirow[b]{2}{*}{$P$ value } \\
\hline & Uncontrolled & $\begin{array}{c}\text { Partly } \\
\text { controlled }\end{array}$ & Controlled & & & Uncontrolled & $\begin{array}{c}\text { Partly } \\
\text { controlled }\end{array}$ & Controlled & & \\
\hline \multicolumn{11}{|l|}{ Class 1} \\
\hline XQLG add-on group & 47 & 5 & 0 & 50.80 & \multirow[b]{2}{*}{0.8292} & 6 & 30 & 16 & 46.67 & \multirow[b]{2}{*}{0.1291} \\
\hline Placebo add-on group & 44 & 4 & 0 & 50.16 & & 5 & 20 & 23 & 54.64 & \\
\hline \multicolumn{11}{|l|}{ Class 2} \\
\hline XQLG add-on group & 29 & 3 & 0 & 36.28 & \multirow{2}{*}{0.5173} & 2 & 15 & 15 & 40.65 & \multirow{2}{*}{0.0329} \\
\hline Placebo add-on group & 36 & 2 & 0 & 34.84 & & 8 & 20 & 10 & 31.15 & \\
\hline
\end{tabular}

(\%) did not appear to impact class membership. Even though there was a trend for amelioration of $\mathrm{FEV}_{1}$ in the XQLG addon group, no significant differences were found. In the subset of 70 patients with the above-mentioned typical symptoms, XQLG add-on therapy was found to significantly increase the levels of asthma control according to GINA guidelines $(P=$ 0.0329) (Table 7). There was no significant difference in the increase in the levels of asthma control between Groups A and $B$ in the remaining subset of 100 patients with relatively low sensitivity of the above-mentioned symptoms $(P=0.1291)$ (Table 7).

4.2. Safety. Overall, treatment with XQLG and seretide was well tolerated. Neither group showed abnormal findings in hematology, or serum chemistry tests. No serious adverse effects were observed in either group. In particular, there were no deaths, hospitalizations, or emergency room visits. A total of one AE was reported by one patient $(0.9 \%)$ in the XQLG add-on group with mild sinus tachycardia, which was of mild intensity, did not necessitate treatment discontinuation, and was not deemed to be treatment related by the investigator.

\section{Discussion}

The last decade has seen an increased interest in the use of TCM for asthma treatment, and effects have been documented in numerous conditions. Since 2005, several controlled clinical studies of "antiasthma" herbal remedies have been published in SCI indexed journals $[49,50]$. XQLD has played an important role in preventing and treating lung diseases in China and other Asian countries for centuries where it is still used as a monotherapy or in integrated medicine. Such a wide spectrum of clinical efficacy suggests that the power of XQLG add-on therapy may help restore lung function. However, well-controlled clinical trials using herbs for the treatment of asthma are rare.

Using our randomized, double-blind, positive controlled, parallel-group design, we demonstrate that XQLG add-on therapy to seretide can significantly increase baseline levels of asthma control according to GINA guidelines ACT score, asthma symptoms score, $\mathrm{FEV}_{1}$, and PEF in asthmatic subjects. However, there were no differences between XQLG and control groups at the 90-days assessment. These findings are not completely consistent with the results of previous

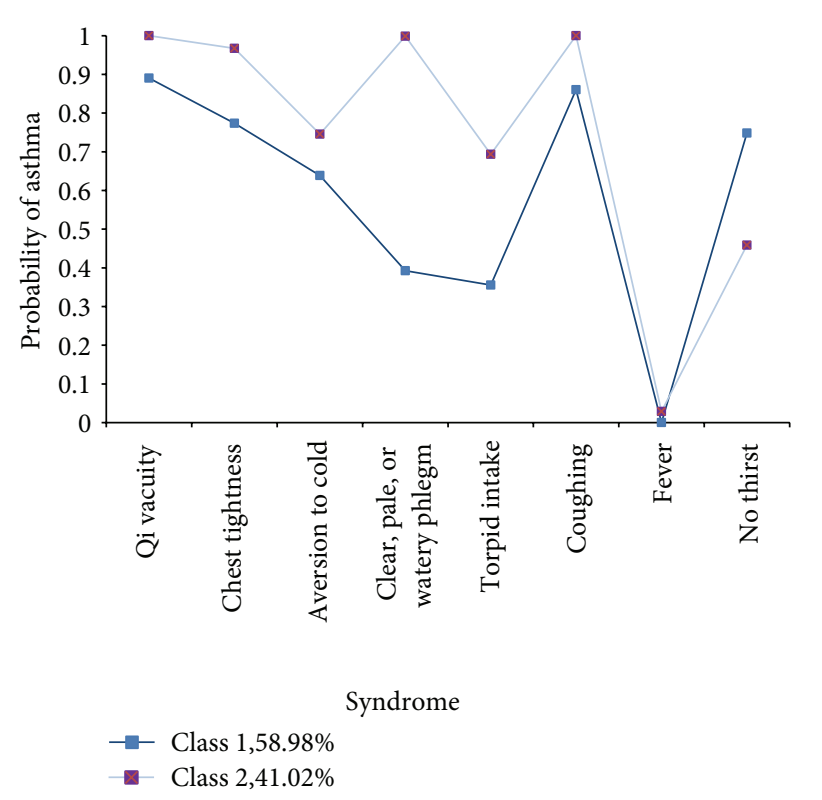

FIgURE 3: Conditional probabilities for each item endorsement according to latent class for asthma participants in subjects $(n=$ 170).

trials [13]. According to the published literature, some trials have identified significant differences in responses between XQLD add-on therapy and conventional western medicines although both of them were obviously better than baseline. Additionally, $\mathrm{FEV}_{1}$ and $\mathrm{PEF}$ were significantly better than conventional western medicines groups. In contrast, the data we obtained in the current study showed no clinically significant difference in the above parameters when XQLG was added to seretide, compared to when placebo was added. Thus it is necessary to include precise traditional criteria into the inclusion criteria of clinical studies on traditional herbal combinations.

The associations observed in this study must be viewed with a number of considerations. A possible interpretation of no clinically significant differences for the overall control of asthma may not take into account that certain specific characteristics of asthma control may in fact show clinically significant improvements, some of which may not be primary endpoints and may impact quality of life, such as days where normal activity is limited due to asthma symptoms, or visits 
to the emergency department or hospital. Some of these are included in the evaluation of a total asthma control symptom score, but these scores are frequently not broken down in clinical trials. Moreover, analysis of the entire group as a whole may not take into consideration that some patients with certain symptom patterns may in fact show a higher level of improvement if analyzed separately. A larger study will allow for stratification of patients into different groups based on age, socioeconomic status, gender, and so forth. In our case, screening patients with symptoms that CM identifies as important in asthma status may help to identify response to specific treatments in specific groups. The magnitude of pattern effect in a TCM clinical trial is often calculated as the benefit in the TCM arm relative to the benefit in the control arm. According to the Chinese Pharmacopoeia (2005 edition) [12], indications of XQLG include wind-coldretained fluid pattern manifested as aversion to cold, fever, absence of sweating, wheezing, and cough with watery phlegm. In fact, our study demonstrates that if those patients with TCM symptoms can be separated out using latent class analysis, the effect of XQLG may be statistically significant when compared to placebo in this study.

Comparative latent class analysis is a statistical method to define symptom patterns for the purposes of distinguishing certain patient groups. This method was used in this study to analyze in greater depth the efficacy of the XQLG treatments. The determination of what treatment is best for what kinds of patients is a general objective of clinical research [51]. One major factor is the application of the LCA in an effort to determine which treatment is preferable for which kind of patients. Interestingly, results indicate that all the subjects can be classified into two distinct subsets according to the conditional probabilities of asthma participants found in the LCA. The symptom trend was similar in both classes, but there were clear differences. The subtype of 70 participants had a higher probability of reporting adherence to the six typical symptoms, including Qi vacuity, chest tightness, aversion to cold, clear, pale, or watery phlegm, torpid intake, and coughing. When this group was analyzed separately for the comparative efficacy of XQLG versus placebo add-on therapy, significantly increased levels of asthma control were found in the former group $(P=0.0329)$ [9]. The implications of this finding is that one may be able to use TCM symptom pattern recognition to study and subsequently identify in clinical practice those patients who may respond optimally to a specific herbal treatment.

There were no serious adverse events and side effects were insignificant, in line with other similar studies [13]. XQLG intervention appears to be a safe mode of therapy in our patient study group. However, in the United States, XQLD is not available from a pharmacy or via a doctor's prescription because this formula includes an illegal herb ephedra [52]. New evidence indicates that rational use can avoid the toxicity of ephedra [52]. It is important to note that ephedra must always be prescribed with care and the safe use of a combination of western medical remedies with herbal therapy should result from the evaluation of drug-herb interactions.

We strictly defined TCM indications in inclusion criteria. All subjects were characterized as TCM cold pattern by using the strict predefined criteria. However, we almost certainly underestimated pattern classification accuracy by using current criteria. If other TCM classifications of asthma are included, the results may be different $[53,54]$.

This study has other limitations. First, our subtype population was relatively small (class $1=100$; class $2=70$ ). Second, the use of multiple single-item symptom measures raises questions regarding reliability and validity. In the design of clinical trials on herbal remedies, a selection of only a few of the multitude of symptoms that asthma patients experience are evaluated. In this study, "asthma of cold pattern" according to current criteria of diagnosis and therapeutic effect of disease and syndromes and the diagnosis mild-to-moderate asthma might not suffice.

Despite these limitations, this study makes an important contribution to impact assessment of symptoms on the treatment efficacy in asthma patients. As we look toward future generations of TCM asthma trials, the concept of individualized therapy based on predictive symptom markers to better select patients who would benefit from a specific intervention is likely to be integral. A recent perspective on considering inclusion and exclusion criteria for randomized controlled trials (RCTs) may integrate genotype through characteristic symptoms [55]. It shows how meaningful stratification based on symptomatology may be, and how careful selection of patients for targeted therapies may be of utmost importance for developing future treatment strategies.

Further assessment is warranted of the role of XQLG in adult asthmatic patients with six TCM typical symptoms. If reproducible, these results provide support for designing interventions aimed at more definite symptoms in the millions of patients currently treated for asthma.

\section{Conclusions}

The addition of XQLG to existing treatment using combination inhaled corticosteroid/long acting beta-agonist (LABA) therapy did not demonstrate a statistically significant improvement over placebo add-on therapy. However, latent class analysis of Chinese medicine symptom patterns was able to define a subset of patients who would respond to XQLG add-on therapy significantly better than placebo. XQLG may therefore be effective in the treatment of a subgroup of asthma patients and additional studies should be conducted to investigate efficacy and safety of this herbal medication as first line therapy for asthma. This study also illustrates the importance of elucidating ways of defining selective patient populations with favorable response to specific treatment modalities.
Abbreviations
ACT: Asthma control test
AE: Adverse event
CAM: Complementary or alternative medicine
CHM: Chinese herbal medicine
GATPC: The guideline of asthma treatment and prevention in China 
H1R: Histamine $\mathrm{H}(1)$ receptor

HDC: Histidine decarboxylase

ITT: Intent-to treat

IVR: Interactive voice response

IWR: Interactive web response

LCA: Latent class analysis

RCT: Randomized controlled trial

SAE: Serious adverse event

SCRT: So-cheong-ryong-tang

SR: Systematic review

SST: Sho-seiryu-to

TDI: Toluene-2,4-diisocyanate

XQLD: Xiaoqinglong decoction

XQLG: Xiaoqinglong granules.

\section{Conflict of Interests}

The authors declare that they have no conflict of interests.

\section{Authors' Contribution}

Q. Zha, S. Lin, and C. Zhang contributed equally to this work.

\section{Acknowledgments}

This study is supported by The National Eleventh Five-Year Support Project of China (2006BAI08B02-2). This research is partly sponsored by the projects from the National Science Foundation of China (30825047).

\section{References}

[1] C. Chang, "Asthma in children and adolescents: a comprehensive approach to diagnosis and management," Clinical Reviews in Allergy and Immunology, pp. 1-40, 2011.

[2] S. Louie, B. M. Morrissey, N. J. Kenyon, T. E. Albertson, and M. Avdalovic, "The critically Ill asthmatic-from ICU to discharge," Clinical Reviews in Allergy and Immunology, pp. 1-15, 2011.

[3] A. A. Zeki, N. J. Kenyon, K. Yoneda, and S. Louie, "The adult asthmatic," Clinical Reviews in Allergy and Immunology, pp. 118, 2011.

[4] R. R. Vatti and S. S. Teuber, "Asthma and pregnancy," Clinical Reviews in Allergy and Immunology, vol. 43, no. 1-2, pp. 45-56, 2012.

[5] C. E. Clark, E. Arnold, T. J. Lasserson, and T. Wu, "Herbal interventions for chronic asthma in adults and children: a systematic review and meta-analysis," Primary Care Respiratory Journal, vol. 19, no. 4, pp. 307-314, 2010.

[6] G. Passalacqua, P. J. Bousquet, K. H. Carlsen et al., "ARIA update: I-systematic review of complementary and alternative medicine for rhinitis and asthma," The Journal of Allergy and Clinical Immunology, vol. 117, no. 5, pp. 1054-1062, 2006.

[7] X. M. Li, "Traditional Chinese herbal remedies for asthma and food allergy," The Journal of Allergy and Clinical Immunology, vol. 120, no. 1, pp. 25-31, 2007.

[8] F. P. Chen, F. J. Chen, M. S. Jong, H. L. Tsai, J. R. Wang, and S. J. Hwang, "Modern use of Chinese herbal formulae from ShangHan Lun," Chinese Medical Journal, vol. 122, no. 16, pp. 18891894, 2009.
[9] Z. Zhang, C. Mitchell, Y. Feng, and N. Wiseman, Shang Hán Lùn, Paradigm Publications, 1999.

[10] B. Flaws and P. Sionneau, The Treatment of Modern Western Medical Diseases with Chinese Medicine: A Textbook \& Clinical Manual, Blue Poppy Press, 2001.

[11] P. Wang, Zhong Yi Shi Da Ming Fang, Zhongguo Zhong Yi Yao Chu Ban She, Beijing, China, 1998.

[12] China Pharmacopoeia Commission, Pharmacopoeia of the People's Republic of China, Chemical lndustry Press, 2005.

[13] H. L. Yang, H. L. J. P, Y. H. Li, and M. Z. Guo, "A system evaluation on randomized control trials: xiaoqinglong decoction in treatment of asthma," Journal of Beijing University of Traditional Chinese Medicine, vol. 15, pp. 25-29, 2008.

[14] X. J. Xiong and J. Wang, "Discussion of related problems in herbal prescription science based on objective indications of herbs," Journal of Chinese Integrative Medicine, vol. 8, no. 1, pp. 20-24, 2010.

[15] J. S. Byun, S. Y. Yang, I. C. Jeong et al., "Effects of So-CheongRyong-Tang and Yeon-gyo-pae-dok-san on the common cold: randomized, double blind, placebo controlled trial," Journal of Ethnopharmacology, vol. 133, no. 2, pp. 642-646, 2011.

[16] E. Ko, S. Rho, C. Cho et al., "So-Cheong-Ryong-Tang, tradititional Korean medicine, suppresses Th2 lineage development," Biological and Pharmaceutical Bulletin, vol. 27, no. 5, pp. 739743, 2004.

[17] Y. Y. Kung, Y. C. Chen, S. J. Hwang, T. J. Chen, and F. P. Chen, "The prescriptions frequencies and patterns of Chinese herbal medicine for allergic rhinitis in Taiwan," Allergy, vol. 61, no. 11, pp. 1316-1318, 2006.

[18] Y. Takeuchi, Y. Nishimura, and T. Yoshikawa, "A comparison between Chinese blended medicine"Shoseiryuto" tranilast and ketotifen on the anti-allergic action in the guinea pigs," Japanese Journal of Allergology, vol. 34, no. 6, pp. 387-393, 1985.

[19] H. Yamada, T. Nagai, M. Nakao et al., "Proteomic analysis of anti-inflammatory effects of a kampo (Japanese Herbal) medicine "shoseiryuto (Xiao-Qing-Long-Tang)" on airway inflammation in a mouse model," Evidence-based Complementary and Alternative Medicine, vol. 2011, Article ID 604196, 13 pages, 2011.

[20] S. Amagaya, A. Iizuka, B. Makino et al., "General pharmacological properties of Sho-seiryu-to (TJ-19) extracts," Phytomedicine, vol. 8, no. 5, pp. 338-347, 2001.

[21] T. Nagai, Y. Arai, M. Emori et al., "Anti-allergic activity of a Kampo (Japanese herbal) medicine "Sho-seiryu-to (XiaoQing-Long-Tang)" on airway inflammation in a mouse model," International Immunopharmacology, vol. 4, no. 10-11, pp. 13531365, 2004.

[22] Y. Tanno, Y. Shindoh, and T. Takishima, "Modulation of human basophil growth in vitro by Xiao-Qing-Long-Tang (Syoseiryu-to), Chai-Pu-Tang (Saiboku-to), Qing-Fei-Tang (Seihaito), baicaalein and ketotifen," American Journal of Chinese Medicine, vol. 17, no. 1-2, pp. 45-50, 1989.

[23] A. K. Nyunt, Y. Takeuchi, K. Yokomuro, and Y. Miyanaga, "Comparative studies on the antiallergic effects of kampo medicines used for the therapy of respiratory diseases," Japanese Journal of Allergology, vol. 44, no. 4, pp. 503-512, 1995.

[24] M. Sakaguchi, A. Iizuka, M. Yuzurihara et al., "Pharmacological characteristics of Sho-seiryu-to, an antiallergic Kampo medicine without effects on histamine $\mathrm{H} 1$ receptors and muscarinic cholinergic system in the brain," Methods and Findings in Experimental and Clinical Pharmacology, vol. 18, no. 1, pp. 4147, 1996. 
[25] S. T. Kao, C. S. Lin, C. C. Hsieh, W. T. Hsieh, and J. G. Lin, "Effects of xiao-qing-long-tang (XQLT) on bronchoconstriction and airway eosinophil infiltration in ovalbumin-sensitized guinea pigs: in vivo and in vitro studies," Allergy, vol. 56, no. 12, pp. 1164-1171, 2001.

[26] H. M. Kirn, G. S. Yoon, J. U. Seo, G. Moon, H. R. Kirn, and T. Y. Shin, "Inhibition of mast cell-mediated anaphylaxis by Sochungryong-Tang," American Journal of Chinese Medicine, vol. 28, no. 1, pp. 69-76, 2000.

[27] J. R. Velazquez and L. M. Teran, "Chemokines and their receptors in the allergic airway inflammatory process," Clinical Reviews in Allergy and Immunology, vol. 41, no. 1, pp. 76-88, 2011.

[28] S. T. Kao, S. D. Wang, L. J. Lin et al., "Xiao-Qing-LongTang attenuates allergic airway inflammation and remodeling in repetitive Dermatogoides pteronyssinus challenged chronic asthmatic mice model," Journal of Ethnopharmacology, vol. 142, no. 2, pp. 531-538, 2012.

[29] J. Kim, S. Natarajan, H. Bae, S. K. Jung, W. Cruikshank, and D. G. Remick, "Herbal medicine treatment reduces inflammation in a murine model of cockroach allergeninduced asthma," Annals of Allergy, Asthma and Immunology, vol. 107, no. 2, pp. 154-162, 2011.

[30] T. Makino, T. Inagaki, K. I. Komatsu, and Y. Kano, "Pharmacokinetic interactions between Japanese traditional medicine (kampo) and modern medicine (III). Effect of Sho-seiryu-to on the pharmacokinetics of azelastine hydrochloride in rats," Biological and Pharmaceutical Bulletin, vol. 27, no. 5, pp. 670673, 2004.

[31] T. Makino, F. Mizuno, and H. Mizukami, "Does a kampo medicine containing schisandra fruit affect pharmacokinetics of nifedipine like grapefruit juice?" Biological and Pharmaceutical Bulletin, vol. 29, no. 10, pp. 2065-2069, 2006.

[32] T. Shimada, M. Kondoh, C. Motonaga et al., "Enhancement of Anti-Allergic effects mediated by the kampo medicine shoseiryuto (Xiao-Qing-Long-Tang in Chinese) with lysed enterococcus faecalis FK-23 in mice," Asian Pacific Journal of Allergy and Immunology, vol. 28, no. 1, pp. 59-66, 2010.

[33] Y. Ikeda, A. Iizuka, S. Amagaya, A. Ishige, Y. Komatsu, and E. Fujihira, "Anti-type I allergic mechanisms of mao-bushisaishin-to in mice," Japanese Journal of Pharmacology, vol. 82, no. 1, pp. 29-33, 2000.

[34] A. Yafune and J. C. Cyong, "Population pharmacokinetic analysis of ephedrine in Kampo prescriptions: a study in healthy volunteers and clinical use of the pharmacokinetic results," International Journal of Clinical Pharmacology Research, vol. 21, no. 2, pp. 95-102, 2001.

[35] K. H. Chan, M. C. Hsu, F. A. Chen, and K. F. Hsu, "Elimination of ephedrines in urine following administration of a Sho-seiryuto preparation," Journal of Analytical Toxicology, vol. 33, no. 3, pp. 162-166, 2009.

[36] J. W. Chen, "A study on the quality of life for asthma patients with Xiao Qing Long Tang," Fujian Journal of Traditional Chinese Medicine, vol. 42, pp. 13-14, 2011.

[37] E. Brand, A Clinician's Guide to Using Granule Extracts, Blue Poppy Press, 2010.

[38] State Food and Drug Administration of China, "Supplementary Rules for TCM Registration," http://www.sfda.gov.cn/.

[39] State Food and Drug Administration of China, "Overview on Registration Administration of Traditional Chinese Medicines (TCM) in China," http://www.who.int/medicines/areas/
quality_safety/regulation_legislation/icdra/4_China_RevisingRegulatoryFramework-HerbalMedicines.pdf.

[40] National Institutes of Health, "Guidelines for the Diagnosis and Management of Asthma," National Institutes of Health National Heart, Lung and Blood Institute, http://www .nhlbi.nih.gov/guidelines/asthma/asthsumm.pdf.

[41] "Global Strategy for Asthma Management and Prevention, Global Initiative for Asthma (GINA) 2006," http://www .ginasthma.org/.

[42] State Administration of Traditional Chinese Medicine of the People's Republic of China, Criteria of Diagnosis and Therapeutic Effect of Disease and Syndromes in Traditional Chinese Medicine, Nanjing University Press, Nanjing, China, 1994.

[43] Asthma Group of Chinese Society of Respiratory Diseases, "The guideline of asthma treatment and prevention," Chinese Journal of Tuberculosis and Respiratory Diseases, vol. 31, no. 3, pp. 132138, 2003.

[44] B. W. Liu, B. W. T, C. Yao et al., "The central randomization system in multi-center clinical trials," Chinese Journal of New Drugs and Clinical Remedies, vol. 25, pp. 931-935, 2006.

[45] E. D. Bateman, S. S. Hurd, P. J. Barnes et al., "Global strategy for asthma management and prevention: GINA executive summary," European Respiratory Journal, vol. 31, no. 1, pp. 143-178, 2008.

[46] R. A. Nathan, C. A. Sorkness, M. Kosinski et al., "Development of the asthma control test: a survey for assessing asthma control," The Journal of Allergy and Clinical Immunology, vol. 113, no. 1, pp. 59-65, 2004.

[47] M. Schatz, C. A. Sorkness, J. T. Li et al., "Asthma control test: reliability, validity, and responsiveness in patients not previously followed by asthma specialists," The Journal of Allergy and Clinical Immunology, vol. 117, no. 3, pp. 549-556, 2006.

[48] S. T. Lanza, L. M. Collins, D. R. Lemmon, and J. L. Schafer, "PROC LCA: a SAS procedure for latent class analysis," Structural Equation Modeling, vol. 14, no. 4, pp. 671-694, 2007.

[49] X. M. Li, “Treatment of asthma and food allergy with herbal interventions from traditional chinese medicine," The Mount Sinai Journal of Medicine, vol. 78, pp. 697-716, 2011.

[50] M. C. Wen, C. H. Wei, Z. Q. Hu et al., "Efficacy and tolerability of antiasthma herbal medicine intervention in adult patients with moderate-severe allergic asthma," The Journal of Allergy and Clinical Immunology, vol. 116, no. 3, pp. 517-524, 2005.

[51] R. Simon, "Patient subsets and variation in therapeutic efficacy," British Journal of Clinical Pharmacology, vol. 14, no. 4, pp. 473482, 1982.

[52] C. Wang, B. Cao, Q. Q. Liu et al., “Oseltamivir compared with the Chinese traditional therapy maxingshigan-yinqiaosan in the treatment of H1N1 influenza: a randomized trial," Annals of Internal Medicine, vol. 155, pp. 217-225, 2011.

[53] W. He, Y. Xie, and Y. Wang, "Thought on several problems of clinical revaluationof post-marketing herb research," Zhongguo Zhong Yao Za Zhi, vol. 35, no. 12, pp. 1641-1643, 2010.

[54] W. He, Y. Xie, and Y. Wang, "Postmarketing herbs clinical evaluation should concern about re-evaluating symptoms," Zhongguo Zhong Yao Za Zhi, vol. 35, no. 11, pp. 1498-1500, 2010.

[55] L. Liu, E. L. Leung, and X. Tian, "Perspective: the clinical trial barriers," Nature, vol. 480, no. 7378, p. S100, 2011. 


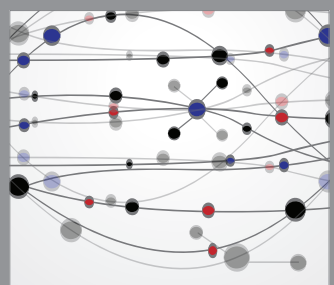

The Scientific World Journal
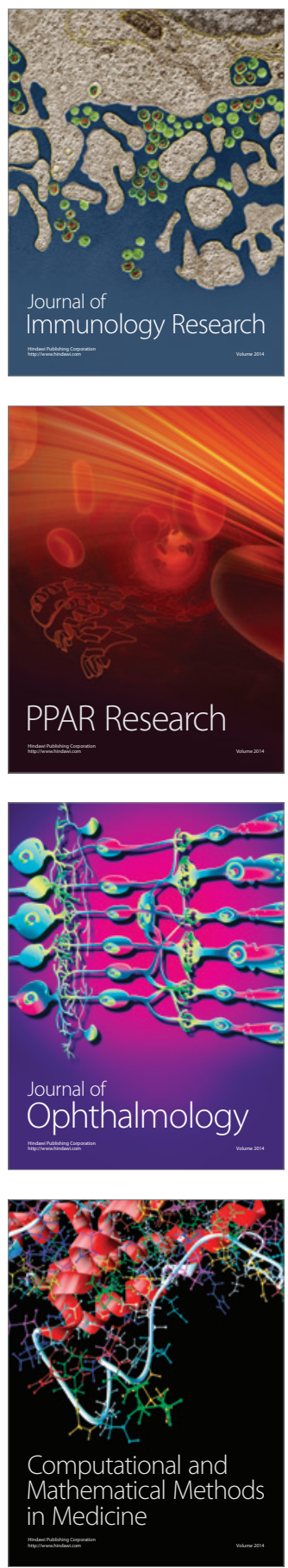

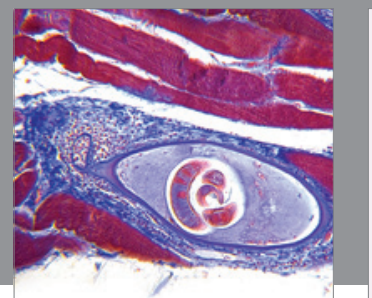

Gastroenterology

Research and Practice
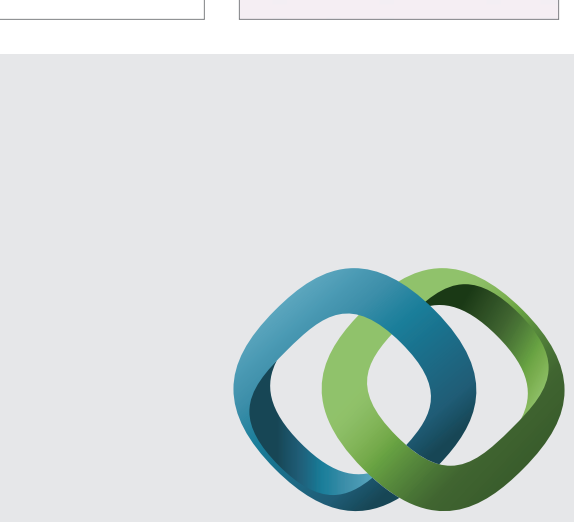

\section{Hindawi}

Submit your manuscripts at

http://www.hindawi.com
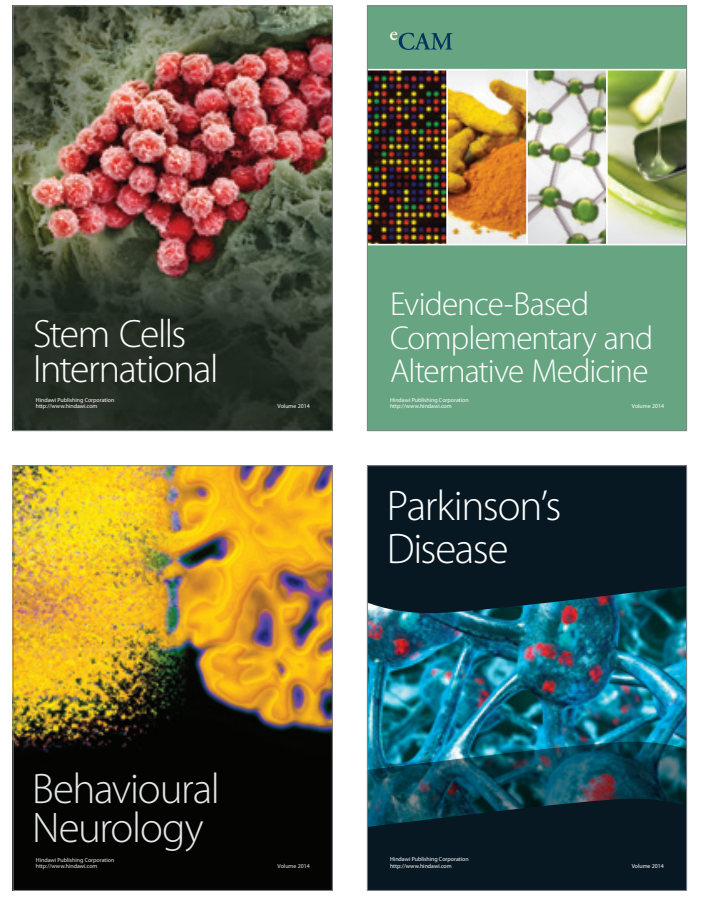
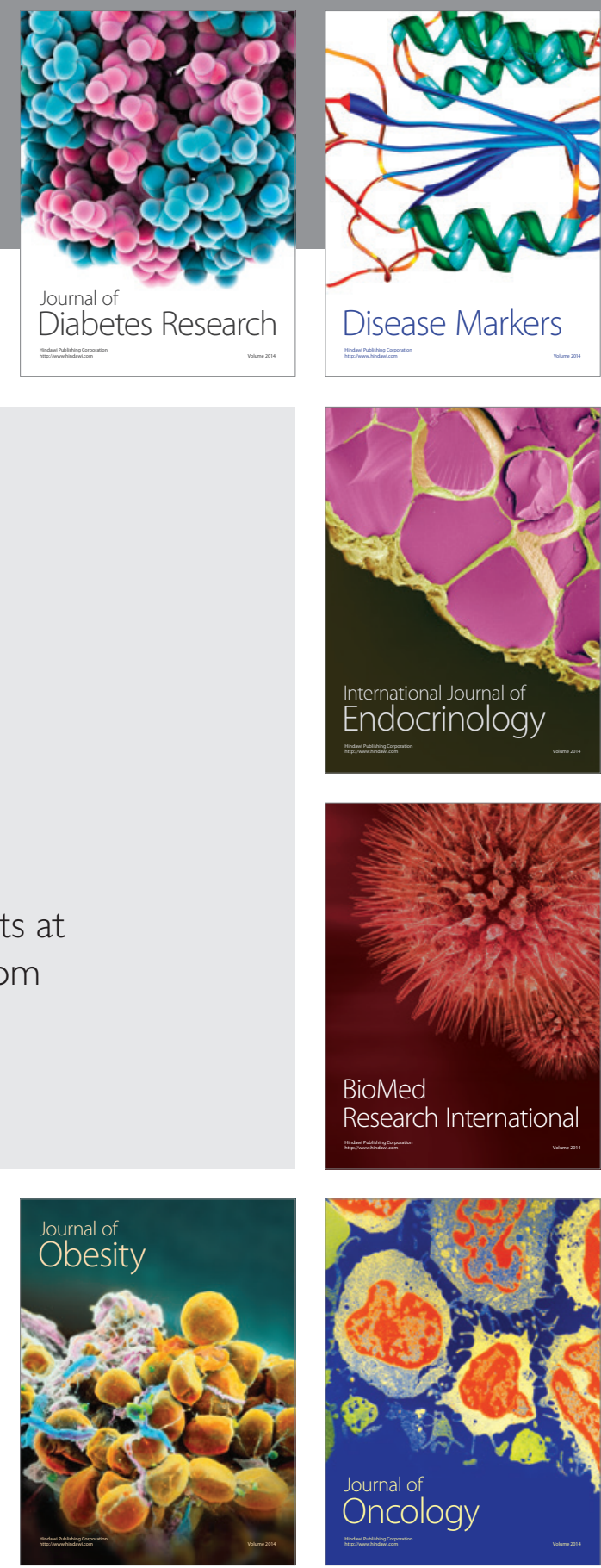

Disease Markers
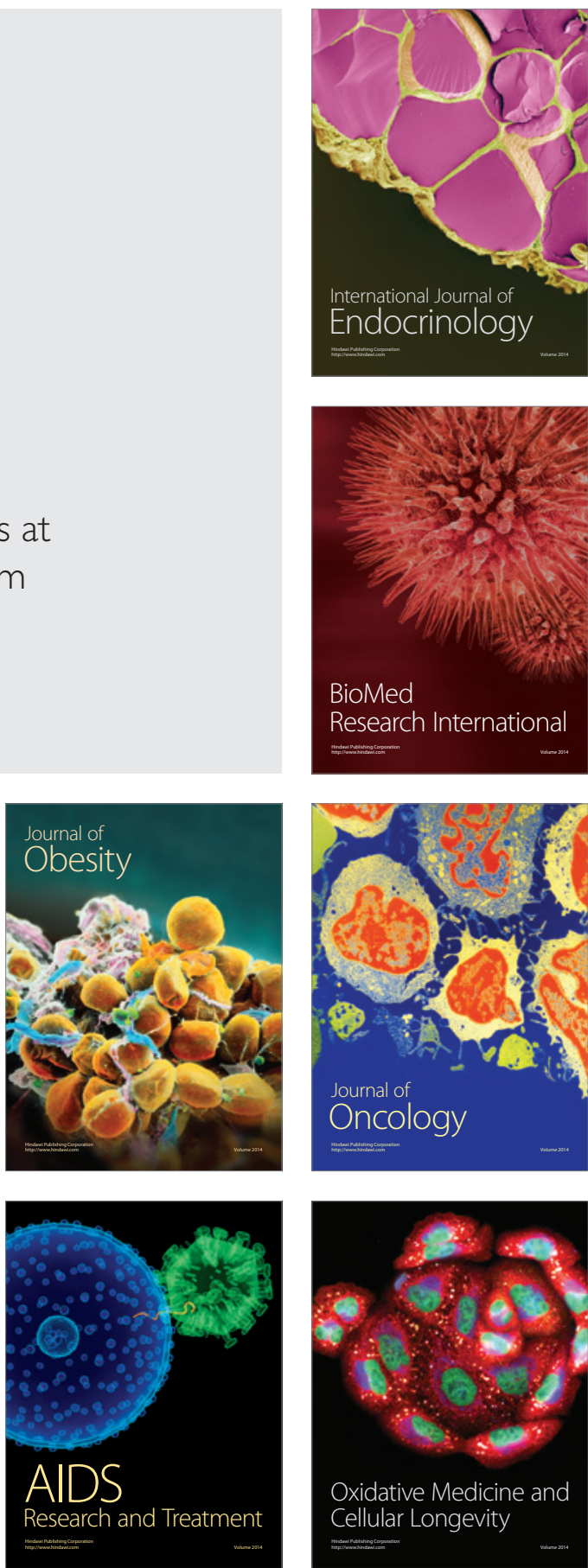